\title{
Ion Selectivities of Some Extracellular Viscous Polysaccharides from Red Algae
}

\author{
Tooru AKahane, * Satoru Kawashima, ${ }^{*}$ Ichiro HiraO, ${ }^{*}$ \\ Toshio SHIMIZU, ${ }^{* *}$ and Akira MINAKATA** \\ *Department of Industrial Chemistry, Numazu College \\ of Technology, Numazu 410, Japan. \\ **Department of Physics, Hamamatsu University School \\ of Medicine, Hamamatsu 431-31, Japan.
}

(Received June 8, 1981)

\begin{abstract}
The potassium and sodium ion selectivities of extracellular polysaccharides in red algae were investigated by an elemental quantitative determination of $\mathrm{Na}^{+}$and $\mathrm{K}^{+}$, the release of these ions in a dialysis experiment, viscosity and potentiometric titration. The elemental analysis indicated that all polysaccharides studied had a higher affinity toward $\mathrm{K}^{+}$than toward $\mathrm{Na}^{+}$in a purified and ion-exchanged state, although only carrageenan had such a tendency in a natural state without purification. The release of $\mathrm{Na}^{+}$from the solution was faster than that of $\mathrm{K}^{+}$for all polysaccharides studied. Among these, $\kappa$-carrageenan released $\mathrm{Na}^{+}$most readily, in agreement with the $\mathrm{K}^{+}$ion selectivity of this polysaccharide. The intrinsic viscosity of the potassium salt was always smaller than that of the sodium salt. Also, viscometry revealed a marked difference in the affinity of $\mathrm{K}^{+}$toward $\kappa$ - and $\lambda$-carrageenans. The potentiometric titration curve, however, showed no difference for either $\mathrm{Na}^{+}$or $\mathrm{K}^{+}$. These results support all cases of $\mathrm{K}^{+}$ion selectivity, especially that of $\kappa$-carrageenan. Possible mechanisms of the difference in the ion specificity among these polysaccharides are discussed.
\end{abstract}

KEY WORDS Polysaccharides / Carrageenan / Ion Selectivity /

Among extracellular viscous polysaccharides in red algae Rhodophyceae, carrageenans have been extensively studied for their polyelectrolyte nature and gel forming properties. ${ }^{1-3}$ In particular, the ion selectivity of carrageenans has been investigated by several methods. ${ }^{4-14}$ However, not many studies have been made on the polyelectrolyte behavior of other species of polysaccharides from Rhodophyceae even though their structure is very similar to that of carrageenans.

Many measurements of the activity of $\mathrm{K}^{+}$and $\mathrm{Na}^{+}$in carrageenan solutions have been made, but the results are in some cases contradictory. For example, Pass et al. $^{11}$ reported that $\gamma_{\mathrm{Na}+}>\gamma_{\mathrm{K}^{+}}$, whereas Podlas and Ander ${ }^{6}$ reported the opposite. The results of Schachat and Morawetz ${ }^{5}$ are $\gamma_{\mathrm{Na}^{+}} \approx$ $\gamma_{\mathrm{K}}-$. Thus, it seems difficult to determine ion selectivity only by measuring the activity of these ions.

To clarify the nature of ion selectivity in carrageenans and other polysaccharides, several ionic properties of these polysaccharides were investigated and compared with each other. In this study, the difference in the affinity of $\mathrm{K}^{+}$and $\mathrm{Na}^{+}$toward polysaccharides was examined by measuring atomic absorption spectra, conductivity, viscosity and by potentiometric titration.

\section{EXPERIMENTAL}

\section{Materials}

Polysaccharides used were obtained by hot water extraction of the following algae; carrageenan from Hypnea japonica, funoran from Gloiopeltis complanata, and two species of agars from Gelidium amansii and Gracilaria verrucosa. These original mucilages were stocked prior to further preparations. Their chemical composition was estimated by the following methods: phenol-sulfuric acid method $^{15}$ for sugar concentration, colloidal titration ${ }^{16}$ for sulfate and carboxyl group content, colorimetry 
T. Akahane et al.

Table I. Analytical data of carrageenan, $\kappa$ - and $\lambda$-carrageenan fractions

\begin{tabular}{|c|c|c|c|c|c|c|}
\hline \multirow{2}{*}{ Sample } & $\mathrm{KCl}$ & Yield & \multirow{2}{*}{$\begin{array}{c}\text { 3,6-Anhydro } \\
\text { galactose }\end{array}$} & \multirow{2}{*}{$\frac{\text { Total acid }}{\mathrm{mol} / \mathrm{galp}^{\mathrm{a}}}$} & \multirow{2}{*}{$\begin{array}{c}\begin{array}{c}\text { Sulphate } \\
\text { groups }\end{array} \\
\text { mol/galp a }^{\text {a }}\end{array}$} & \multirow{2}{*}{$\begin{array}{c}\begin{array}{c}\text { Carboxyl } \\
\text { groups }\end{array} \\
\text { mol/galp }\end{array}$} \\
\hline & $\mathbf{M}$ & $\%$ & & & & \\
\hline \multicolumn{7}{|l|}{ Original } \\
\hline carrageenan & & & 0.47 & 0.45 & 0.41 & 0.04 \\
\hline$\kappa_{1}$ & \multirow{2}{*}{0.1} & 39.7 & 0.47 & 0.35 & 0.35 & 一 \\
\hline$\lambda_{1}$ & & 32.1 & 0.42 & 0.57 & 0.52 & 0.05 \\
\hline$\kappa_{2}$ & \multirow{2}{*}{0.2} & 49.2 & 0.49 & 0.25 & 0.29 & - \\
\hline$\lambda_{2}$ & & 13.8 & 0.42 & 0.54 & 0.52 & 0.02 \\
\hline$\kappa_{3}$ & \multirow{2}{*}{0.3} & 56.0 & 0.49 & 0.37 & 0.33 & 0.04 \\
\hline$\lambda_{3}$ & & 4.0 & 0.45 & 0.57 & 0.52 & 0.05 \\
\hline
\end{tabular}

${ }^{a} \mathrm{~mol} / \mathrm{galp}$, number of groups per galactopyranose unit.

by cystein-sulfuric acid ${ }^{17}$ for 3,6-anhydrogalactose (3,6 AnG), and atomic absorption analysis for $\mathrm{K}^{+}$ and $\mathrm{Na}^{+}$.

Separation of carrageenan into its $\kappa$ - and $\lambda$ fractions was carried out by adding $\mathrm{KCl}$ to a $0.025 \%$ original solution, resulting in a solution $(\lambda)-$ gel $(\kappa)$ phase separation..$^{3,4,18-22}$ The concentration of added $\mathrm{KCl}$ was chosen as $0.1,0.2$ and $0.3 \mathrm{M}$, respectively. ${ }^{18,22}$ Yields of the $\kappa$ - and $\lambda$-fractions and their chemical composition are summarized in Table I. The results indicate that with increasing concentration of $\mathrm{K}^{+}$, the $\kappa$-fraction increased while $\lambda$-fraction decreased. This may have possibly been due to the coprecipitation of the $\lambda$-fraction in the concentrated $\mathrm{KCl}$ solutions. Therefore, according to the results of Cerezo ${ }^{18}$ and of Pernas et al. ${ }^{22} \kappa_{1}$ and $\lambda_{3}$ (hereafter denoted as $\kappa$-Car and $\lambda$-Car, respectively) were used as specimens of the $\kappa$ - and $\lambda$ fractions for further study. These two specimens were also identified as the $\kappa$ - and $\lambda$-carrageenans reported in the literature ${ }^{23}$ on the basis of 3,6 AnG content measurement and ir spectra. That is, $\kappa$-Car showed a very strong ir absorption at $840 \mathrm{~cm}^{-1}$ arising from an axial stretching vibration of a sulphate group, and a very weak absorption at 810 $\mathrm{cm}^{-1}$ due to an equatorial stretching vibration of a sulphate group. In the case of $\lambda$-Car, however, the absorption at $840 \mathrm{~cm}^{-1}$ was weak and that at 810 $\mathrm{cm}^{-1}$ was strong.

Ion exchange of the polysaccharides was carried out as follows. $200 \mathrm{ml}$ of $0.1 \%$ polysaccharide solution was mixed with Amberlite IR-120B cation exchange resins, and stirred for $1 \mathrm{~h}$ at $5^{\circ} \mathrm{C}$. The resins and other contaminants were removed by a
G-1 glass filter and then by a G-3 glass filter. Ion exchange by a column method was avoided because of the rapid degradation of the polysaccharides. After filtration, the solution was immediately mixed with $0.2 \mathrm{~N} \mathrm{NaOH}$ or $0.2 \mathrm{~N} \mathrm{KOH}$ solution to obtain the Na- or $\mathrm{K}$-form of the polysaccharides. The solution was then concentrated by ultrafiltration, precipitated and washed with ethanol, purified with acetone, and freeze dried. The flow chart of the above mentioned preparation procedure is illustrated in Figure 1.

Other chemicals were of reagent grade and used without further purification. The water used was deionized and then distilled.

\section{Methods}

The amounts of $\mathrm{K}^{+}$and $\mathrm{Na}^{+}$in the neutralized and purified polysaccharide samples were determined by a Seiko SAS-722 atomic absorption spectrometer, in which the sample solutions were diluted to the proper concentration for making measurement.

Rate of release of counterions was measured as follows. $1 \mathrm{ml}$ of $0.02 \mathrm{~N} \mathrm{NaCl}$ or $\mathrm{KCl}$ was mixed with $5 \mathrm{ml}$ of the $0.05 \%$ Na-type or K-type sample solution, respectively. After incubation overnight, the solution was dialyzed with a Visking tube against pure water in a $200 \mathrm{ml}$ polypropylene flask to avoid release of metal ions from the glass wall. The outer solution was changed after $1 \mathrm{~h}$, and then every two hours. The amount of released ions in the outer solution was measured by a Seiko SAS-722 atomic absorption spectrometer. The conductivity of the outer solution was measured each hour by a 


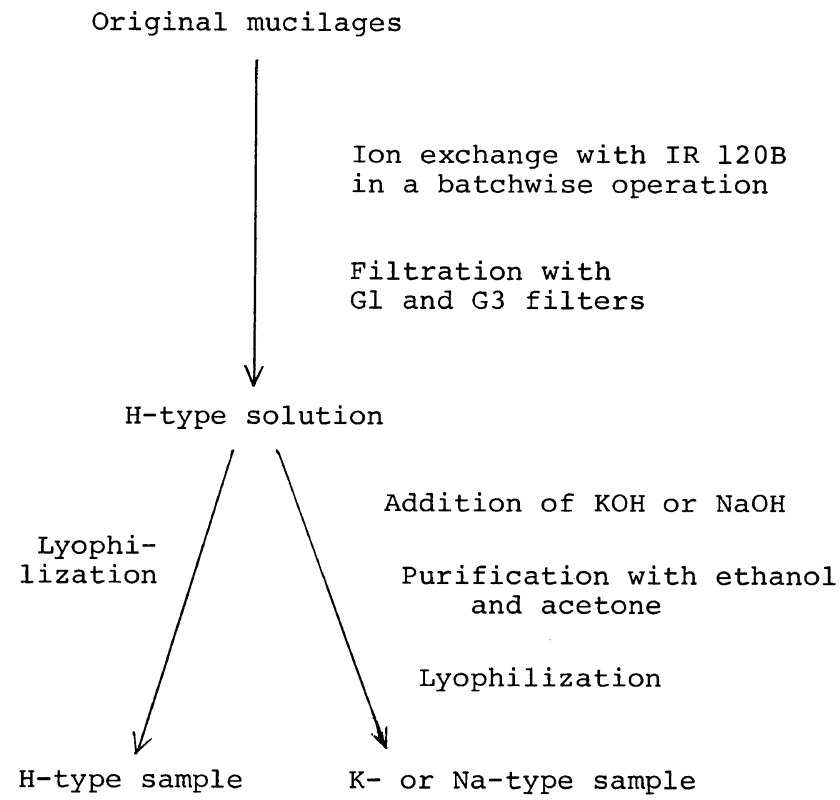

Figure 1. Flow chart for the preparation procedure of H-, K-, and Na-type polysaccharides.

Radiometer CDM-2e conductivity meter, and the change in the conductance was recorded. In this case, the outer solution was changed each hour.

The viscosity was measured with an Ubbelohde type viscometer at $45 \pm 0.05^{\circ} \mathrm{C}$. The solution was prepared so as to contain $0.1 \%$ polysaccharide, $6 \mathrm{M}$ urea and $0.05 \mathrm{M} \mathrm{NaH}_{2} \mathrm{PO}_{4}$ and diluted with the solvent containing $6 \mathrm{M}$ urea and $0.05 \mathrm{M} \mathrm{NaH}_{2} \mathrm{PO}_{4}$. Urea was used to avoid gelation of the polysaccharides. For the same purpose, the solvent containing $0.1 \mathrm{M} \mathrm{NaBr}$ and $0.4 \mathrm{M} \mathrm{Na}$-salicylate (Solv $\mathrm{Na}$ ) was also used for $\mathrm{Na}$-salt of the polysaccharides.

Potentiometric titration was carried out for $20 \mathrm{ml}$ of $3.65 \times 10^{-4} N \kappa$-Car and $3.25 \times 10^{-3} N \lambda$-Car with an Orion $701 \mathrm{~A}$ digital ionalyzer. The titrant, $1.0 \mathrm{~N} \mathrm{NaOH}$ or $1.0 \mathrm{~N} \mathrm{KOH}$ was added to the solution by using a Metrohm E 457 microburet.

To ensure the selectivity of $\mathrm{K}^{+}$in $\kappa$-Car, the following procedure was carried out. First, $\mathrm{H}$-type $\kappa$-Car was titrated with mixture of $0.1 \mathrm{~N} \mathrm{NaOH}$ and $0.1 N \mathrm{KOH}$, and then concentrated by ultrafiltration at room temperature for one day. Secondly, $\mathrm{Na}-\kappa$-Car formed by titration with $0.1 \mathrm{~N} \mathrm{NaOH}$ was dialyzed against a mixture of $1 \mathrm{~N} \mathrm{NaCl}$ and $1 N \mathrm{KCl}$ at $65^{\circ} \mathrm{C}$ for 2 days, and then concentrated by ultrafiltration at room temperature for one day. The
$\mathrm{Na}^{+}$and $\mathrm{K}^{+}$content in both samples was measured by atomic absorption spectroscopy.

\section{RESULTS}

The analytical data for the hot water extracted original samples and $\kappa$ - and $\lambda$-carrageenans are summarized in Table II. Agars and funoran contain roughly the same amounts of alkali metal ions as those of acidic groups, and the content of $\mathrm{K}^{+}$and $\mathrm{Na}^{+}$is almost the same. On the other hand, carrageenan contains about half the amount of alkali metal ions, and the ratio of $\mathrm{K}^{+}$to $\mathrm{Na}^{+}$is about 4 , indicating a low affinity of $\mathrm{Na}^{+}$toward this polysaccharide. $\kappa$-Car and $\lambda$-Car contain more $\mathrm{K}^{+}$than does the original carrageenan, due to the addition of $\mathrm{KCl}$ during the preparation process. The ratio of $\mathrm{K}^{+}$to $\mathrm{Na}^{+}$is almost the same for these two components.

The content of sulphate groups and that of the alkali metal ions in purified $\mathrm{H}-, \mathrm{K}$ - and Na-type polysaccharides are listed in Table III. Evidently, conversion to the $\mathrm{H}$-type results in a loss of the sulphate groups, as anticipated from the low $\mathrm{pH}$ values of the solutions following ion exchange, i.e., 2.9 to 3.2 in every case. Also, measurement of the intrinsic viscosity revealed that the scission of the 
T. AKahane et al.

Table II. Analytical data of original solutions and $\kappa$ - and $\lambda$-carrageenans

\begin{tabular}{|c|c|c|c|c|c|c|}
\hline \multirow{2}{*}{ Sample } & \multirow{2}{*}{$\frac{3,6 \mathrm{AnG}^{\mathrm{a}}}{\%}$} & \multirow{2}{*}{$\frac{\text { Total acid }}{\mathrm{mol} / \mathrm{galp}^{\mathrm{b}}}$} & \multirow{2}{*}{$\frac{\mathrm{SO}_{3}{ }^{-}}{\mathrm{mol} / \text { galp }}$} & \multicolumn{3}{|c|}{ Equiv./equiv. total acid } \\
\hline & & & & $\mathrm{K}^{+}$ & $\mathrm{Na}^{+}$ & $\mathrm{K}^{+}+\mathrm{Na}^{+}$ \\
\hline $\operatorname{Agar}(a)^{c}$ & 41.0 & 0.11 & 0.11 & 0.42 & 0.40 & 0.82 \\
\hline Agar $(v)^{d}$ & 36.5 & 0.26 & 0.26 & 0.42 & 0.45 & 0.87 \\
\hline Funoran & 29.0 & 1.00 & 0.89 & 0.48 & 0.54 & 1.02 \\
\hline Carrageenan & 39.5 & 0.46 & 0.42 & 0.44 & 0.11 & 0.55 \\
\hline$\kappa$-Carrageenan & - & 0.35 & 0.35 & 0.52 & 0.05 & 0.57 \\
\hline$\lambda$-Carrageenan & - & 0.56 & 0.52 & 0.53 & 0.04 & 0.57 \\
\hline
\end{tabular}

a $3,6 \mathrm{AnG}=3,6$-anhydrogalactose.

b $\mathrm{mol} / \mathrm{galp}=$ number of acid groups per galactopyranose unit.

c Agar (a)=extract from Gelidium amansii.

d Agar (v)=extract from Gracilaria verrucosa.

Table III. Analytical data and related properties of H-, K- and Na-type polysaccharides

\begin{tabular}{|c|c|c|c|c|c|c|c|}
\hline \multirow{2}{*}{ Sample } & \multirow{2}{*}{$\frac{\mathrm{SO}_{3}^{-}}{\mathrm{mol} / \text { galp }}$} & \multirow{2}{*}{$\begin{array}{c}(\mathrm{H} / \text { orig. })_{\mathrm{SO}_{3}}{ }^{a} \\
\text { or } \\
(\mathrm{M} / \text { orig. })_{\mathrm{SO}_{3}}{ }^{b}\end{array}$} & \multirow{2}{*}{$\frac{[\eta]}{\mathrm{dlg}^{-1}}$} & \multirow{2}{*}{$(\mathrm{H} / \text { orig. })_{[\eta]^{c}}$} & \multicolumn{3}{|c|}{ Equiv./equiv. total acids } \\
\hline & & & & & $\mathrm{K}^{+}$ & $\mathrm{Na}^{+}$ & $\mathrm{K}^{+}+\mathrm{Na}^{+}$ \\
\hline H-Carrageenan & 0.06 & 0.15 & 0.16 & 0.04 & & & \\
\hline H-Funoran & 0.05 & 0.11 & 0.08 & 0.03 & & & \\
\hline H-Agar (a) & 0.05 & 0.50 & 0.20 & 0.43 & & & \\
\hline H-Agar (v) & 0.07 & 0.18 & 0.17 & 0.08 & & & \\
\hline K-Carrageenan & & & & & 0.91 & 0 & 0.91 \\
\hline $\mathrm{Na}-\mathrm{Carrageenan}$ & 0.40 & 0.95 & & & 0 & 0.57 & 0.57 \\
\hline K-Agar (v) & & & & & 1.05 & 0 & 1.05 \\
\hline Na-Agar (v) & 0.16 & 0.62 & & & 0.02 & 0.59 & 0.61 \\
\hline K-Funoran & 080 & & & & 0.79 & 0.01 & 0.80 \\
\hline Na-Funoran & 0.80 & 0.90 & & & 0.02 & 0.56 & 0.58 \\
\hline
\end{tabular}

${ }^{a}$ (H/orig. $)_{\mathrm{SO}_{3}}$, ratio of $\mathrm{SO}_{3}{ }^{-}$content in $\mathrm{H}$-type to that in original solution.

${ }^{b}$ (M/orig.) $)_{\mathrm{SO}_{3}}$, ratio of $\mathrm{SO}_{3}{ }^{-}$content in $\mathrm{K}$ - or Na-type to that in original solution.

c $(\mathrm{H} / \text { orig. })_{[\eta]}$, ratio of intrinsic viscosity of $\mathrm{H}$-type to that of original solution.

polysaccharide chain occurs during this process, as indicated by the ratio of $[\eta]$ of the $\mathrm{H}$-type and that of the original samples in the table. Considerable loss of the sulphate groups and the chain scission were noted for the polysaccharide containing higher sulphate groups such as funoran. On the other hand, agar from Gelidium amansii (agar (a), the polysaccharide with a low sulphate content) loses only about half its sulphate groups and exhibits a rather low degree of chain scission, i.e., the ratio of $[\eta]$ is about half. Quick neutralization after ion exchange prevents the loss of sulphate groups, as indicated by the small decrease in the content of sulphate groups of the salt form.

K-type polysaccharides including carrageenan contain nearly the same amount of $\mathrm{K}^{+}$as those of acidic groups, in contrast to the case of the original carrageenan containing about half the amount of alkali metal ions. On the other hand, Na-type polysaccharides contain roughly half the amount of $\mathrm{Na}^{+}$. These results support the higher affinity of $\mathrm{K}^{+}$ ion toward all polysaccharides studied. The difference in ion content between the original and purified samples may be ascribed to the difference in conditions; that is, the original samples were contained in a Na-rich medium (sea water), whereas the 


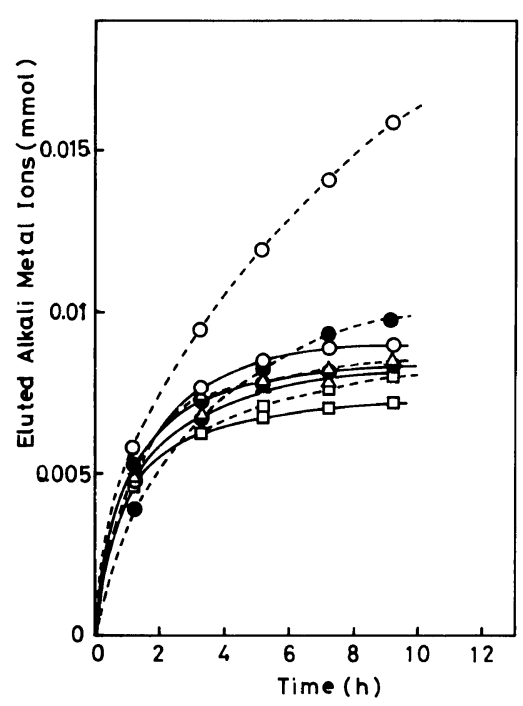

Figure 2. Time course for the elution of alkali metal ions measured by atomic absorption analysis. -- $\mathrm{O}--$, Na-type $\kappa$-Car; -- --, Na-type $\lambda$-Car; $--\triangle_{--}^{-}$, Natype agar (v); -- $\square--$, Na-type funoran; - $\bigcirc-$, K-type $\kappa$-Car; - - - K-type $\lambda$-Car; $-\triangle-$, K-type agar (v); $-\square-, \mathrm{K}$-type funoran.

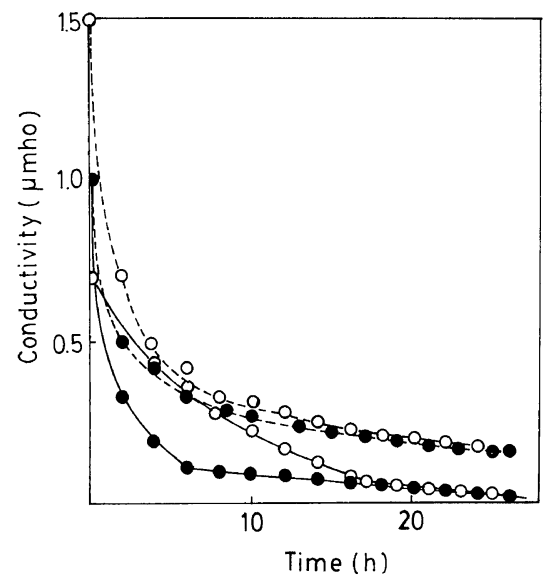

Figure 3. Time course for the conductance of an outer solution at the time of replacement by pure water. $--\mathrm{O}--$, Na-type $\kappa$-Car; --O--, Na-type $\lambda$-Car; -O-, K-type $\kappa$-Car; - - K-type $\lambda$-Car.

K-type and Na-type samples were made artificially.

The time course of the elution of alkali metal ions measured by atomic absorption analysis is plotted in Figure 2. Evidently, the Na-type $\kappa$-Car releases $\mathrm{Na}^{+}$rapidly, compared to other cases. The low affinity of $\mathrm{Na}^{+}$in $\kappa$-Car agrees with the observed selectivity of $\mathrm{K}^{+1,3}$ as will be discussed later. The

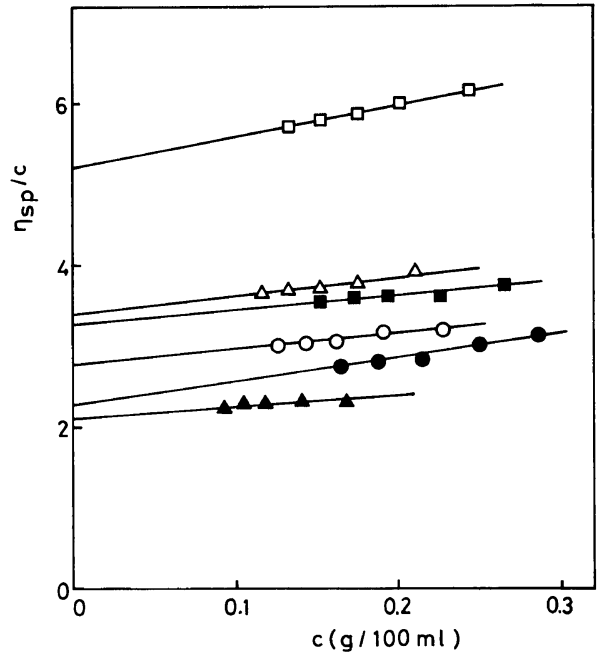

Figure 4. Plots of the reduced viscosity of a solution in the presence of urea against polymer concentration. $\bigcirc$, Na-type carrageenan; $\mathbf{O}$, K-type carrageenan; $\square$, Natype funoran; $\boldsymbol{\square}$, K-type funoran; $\triangle$, Na-type agar (v); $\Delta$, K-type agar (v).

elution of $\mathrm{Na}^{+}$seems slightly faster in each case, although the differences are not so great with other polysaccharides.

Changes in the conductance of the outer solution also indicate the elution rate of counterions. Figure 3 shows the time course for the conductance of an outer solution which was changed each hour. The higher values correspond to the higher elution rates. The elution rates had the following order.

$$
\mathrm{Na}-\kappa \text {-Car }>\mathrm{Na}-\lambda \text {-Car }>\mathrm{K}-\kappa \text {-Car }>\mathrm{K}-\lambda \text {-Car , }
$$

which is consistent with the results shown in Figure 2.

Reduced viscosity of the solution in the presence of urea as an anti-coagulant is plotted against polymer concentration in Figure 4, and related quantities are listed in Table IV. Na-type polysaccharides always exhibited high viscosity. The ratio of $[\eta]$ of Na-type to K-type was smallest for carrageenan, in contrast with the results of counterion release. On the other hand, K-type polysaccharides exhibit higher Huggins' constants which are indicative of intermolecular interaction, that is, a tendency to form aggregates.

The reduced viscosity of $\kappa$-Car was compared 
Table IV. Viscosity of K- and Na-type polysaccharides in the presence of $6 \mathrm{M}$ urea and $0.05 \mathrm{M} \mathrm{NaH}_{2} \mathrm{PO}_{4}$

\begin{tabular}{|c|c|c|c|c|c|c|}
\hline \multirow{2}{*}{$\begin{array}{c}\text { Sample } \\
\text { Type }\end{array}$} & \multicolumn{2}{|c|}{ Carrageenan } & \multicolumn{2}{|c|}{ Agar (v) } & \multicolumn{2}{|c|}{ Funoran } \\
\hline & $\mathrm{K}$ & $\mathrm{Na}$ & $\mathrm{K}$ & $\mathrm{Na}$ & $\mathrm{K}$ & $\mathrm{Na}$ \\
\hline$[\eta]$ & 2.26 & 2.77 & 2.11 & 3.40 & 3.25 & 5.20 \\
\hline$[\eta]_{\mathrm{Na}} /[\eta]_{\mathrm{K}}$ & & 1.23 & & 1.61 & & 1.60 \\
\hline $\mathrm{K}^{\prime}$ & 0.58 & 0.25 & 0.31 & 0.19 & 0.18 & 0.14 \\
\hline
\end{tabular}

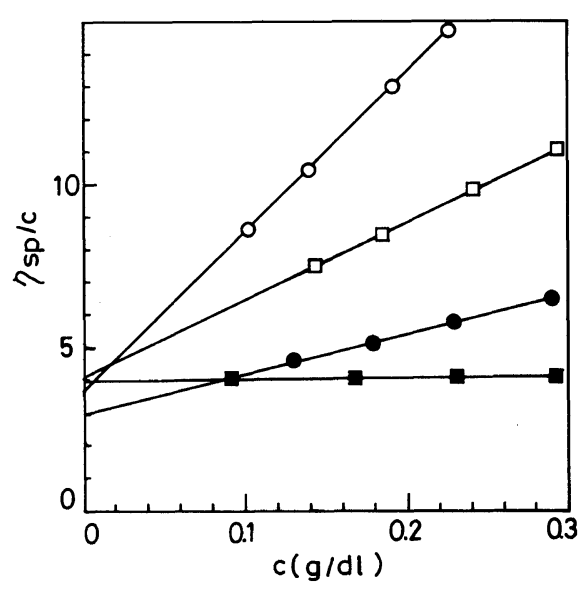

Figure 5. Comparison of concentration dependence of reduced viscosity between $\kappa$ - and $\lambda$-carrageenan in two solvent systems. $\square, \kappa$-Car in $6 \mathrm{M}$ urea; $\square, \lambda$-Car in $6 \mathrm{M}$ urea; $\bigcirc, \kappa$-Car in Solv Na;,$\lambda$-Car in Solv Na.

with that of $\lambda$-Car in two solvent systems, i.e., in the presence of urea and Na-salicylate, respectively. Figure 5 indicates that $\kappa$-Car shows a larger $[\eta]$ value as well as larger concentration dependence than $\lambda$-Car does. Also, this result implies that $\kappa$-Car may have a tendency to aggregate in Na-salicylate solution (Solv $\mathrm{Na}$ ). When $\mathrm{Na}^{+}$and $\mathrm{K}^{+}$are mixed by keeping the total base concentration constant, both $[\eta]$ and Huggins' constant are intermediate between two cases containing only one of these.

Potentiometric titration curves for $\kappa$ - and $\lambda$ carrageenans with $\mathrm{KOH}$ and $\mathrm{NaOH}$ are shown in Figure 6. No appreciable difference could be observed in titration with $\mathrm{KOH}$ or $\mathrm{NaOH}$. This may correspond to the results that the activity coefficients of these ion species are not definitely different from each other, ${ }^{5,14}$ or sometimes contradic-

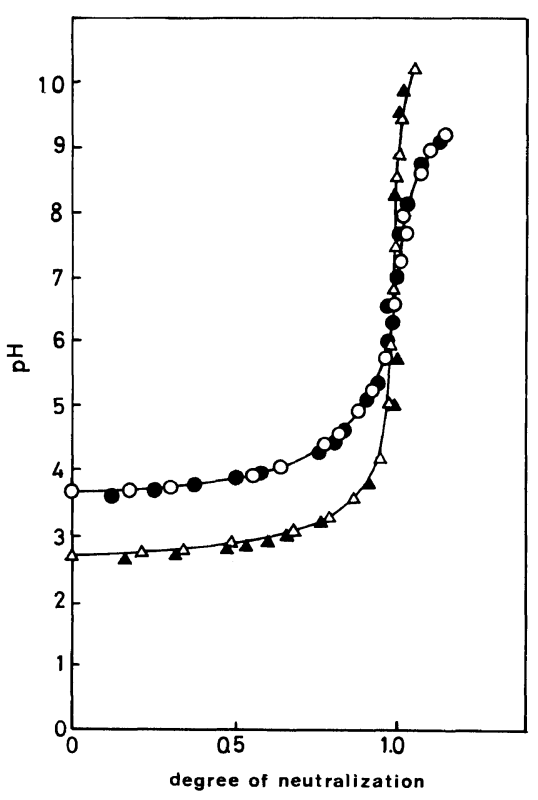

Figure 6. Potentiometric titration curves of $\kappa$ - and $\lambda$ carrageenans with $\mathrm{KOH}$ and with $\mathrm{NaOH}$. $\mathrm{O}, \kappa$-Car with $\mathrm{KOH} ; \triangle, \lambda$-Car with $\mathrm{KOH} ; \boldsymbol{O}, \kappa$-Car with $\mathrm{NaOH}$; $\lambda$-Car with $\mathrm{NaOH}$.

tory. ${ }^{6,11}$ The titration curves of $\kappa$ - and $\lambda$-carrageenan do not coincide with each other since the $\kappa$-carrageenan solution is about 10 times dilute as the $\lambda$-carrageenan solution.

Results of measurement on the selectivity of $\mathrm{K}^{+}$ in $\kappa$-Car are as follows. The ratio of $\mathrm{Na}^{+}$to $\mathrm{K}^{+}$in the titrated sample with a mixture containing the same amounts of $\mathrm{NaOH}$ and $\mathrm{KOH}$ is $37: 63$; that in the dialyzed sample against a mixture of $\mathrm{NaCl}$ and $\mathrm{KCl}$ is $33: 67$. These data indicate the replacement of $\mathrm{Na}^{+}$by $\mathrm{K}^{+}$, which is additional evidence for the selectivity of $\mathrm{K}^{+}$in $\kappa$-Car.

\section{DISCUSSION}

The affinity of $\mathrm{K}^{+}$ions toward all polysaccharides studied is considered to be higher than that of $\mathrm{Na}^{+}$ions, as indicated in Table III. The release of counterions in the dialysis experiments is consistent with the higher affinity of $\mathrm{K}^{+}$ions. Also the lower values of $[\eta]$ of $\mathrm{K}$-type polysaccharides support the above conclusion, i.e., the expansion of the polymer chain may be depressed by the binding of $\mathrm{K}^{+}$ions. Contrary to the values of $[\eta]$, K-type poly- 
saccharides show higher Huggins' constants, suggesting a tendency of aggregation due to a reduction in the overall charge density as a result of $\mathrm{K}^{+}$ion binding. Above all, $\kappa$-carrageenan shows a marked relative selectivity of $\mathrm{K}^{+}$toward $\mathrm{Na}^{+}$, as observed by the release of counterions and the viscosity in urea and salicylate solutions.

This $\mathrm{K}^{+}$ion specificity had already been observed in other polyions containing $\mathrm{SO}_{3}^{-} .{ }^{24}$ Therefore, the specificity may be ascribed primarily to the difference in hydration of ions and sulphate groups. That is, the hydration of a sulphate group is weaker than that of an alkali metal ion, leading to a higher affinity of ions with smaller hydrated radius, i.e. $\mathrm{K}^{+} .{ }^{6,25}$ Other possible factors responsible for the specificity are charge density, distribution of the charged groups and conformation of the polymer chain.

$\mathrm{K}^{+}$ion specificity in $\kappa$-carrageenan may be related to its capability for gel formation, since most of the reasons stated above apply to all polysaccharides studied. $\kappa$-Carrageenan consists of an array of charged groups separated by equal intervals, whereas other polysaccharides consist of an array of a few charged groups situated close together, ${ }^{26}$ as shown in Figure 7. The arrangement of charged groups in $\kappa$-carrageenan may be responsible for the formation of a higher ordered structure. Actually, funoran and $\lambda$-carrageenan are known to take on a random coil in solution, whereas $\kappa$-carrageenan and agars with high 3,6anhydrogalactose content are capable of forming a hydrogen bonded double helical structure. ${ }^{2,3}$ $\kappa$-Carrageenan contains 3,6-anhydrogalactose amounting to nearly $50 \%$ of the total galactopyranose units, thus providing a rigidity of the main chain favorable to helical structure formation. This means that $\kappa$-carrageenan has a low charge density, and is rather hydrophobic, leading to a low affinity to $\mathrm{Na}^{+}$ions, as indicated in Figures 2 and 3 . However, the formation of such a rigid helical structure may provide some spaces for localization of ions with a small hydrated radius such as that of $\mathrm{K}^{+}$ions. These results are consistent with those of Pass et al. ${ }^{11}$ which indicate a higher activity coefficient of $\mathrm{Na}^{+}$than that of $\mathrm{K}^{+}$.

Acknowledgement. The authors are grateful to Professor K. Katsuura for his guidance and suggestions throughout this work.
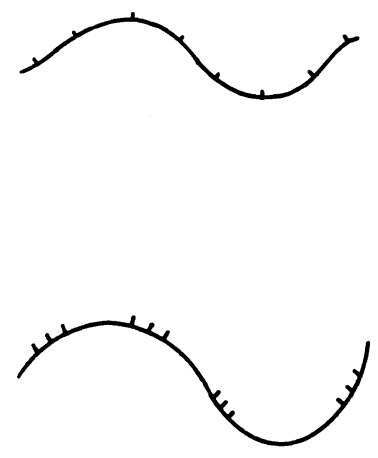

Figure 7. Schematic illustration of distribution of charged groups in the polysaccharides. Upper, $\kappa$-carrageenan; lower, $\lambda$-carrageenan.

\section{REFERENCES}

1. D. B. Smith, W. H. Cook, and J. L. Neal, Arch. Biochem. Biophys., 53, 192 (1953).

2. N. S. Anderson, J. W. Campbell, M. M. Harding, D. A. Rees, and J. W. B. Samuel, J. Mol. Biol., 45, 85 (1969).

3. E. R. Morris, D. A. Rees, and G. Robinson, J. Mol. Biol., 138, 349 (1980).

4. M. E. Zabik and P. J. Aldrich, J. Food Sci., 32, 91 (1967).

5. R. E. Schachat and H. Morawetz, J. Phys. Chem., 61, 1170 (1957).

6. T. J. Podlas and P. Ander, Macromolecules, 2,432 (1969).

7. T. J. Podlas and P. Ander, Macromolecules, 3, 154 (1970).

8. R. E. Nelson and P. Ander, J. Phys. Chem., 75, 1691 (1971).

9. H. Noguchi, K. Gekko, and S. Makino, Macromolecules, 6, 438 (1973).

10. T. H. M. Snoeren and T. A. Payens, Biochim. Biophys. Acta, 437, 264 (1976).

11. G. Pass, G. O. Phillips, and D. J. Wedlock, Macromolecules, 10, 197 (1977).

12. W. R. Ashton, G. Pass, G. O. Phillips, and D. J. Wedlock, J. Chem., Soc., Perkin II, 1229 (1977).

13. M. Kowblansky, M. Tomasula, and P. Ander, $J$. Phys. Chem., 82, 1491 (1978).

14. M. Rinaudo, A. Karimian, and M. Milas, Biopolymers, 18, 1673 (1979).

15. M. Dubois, K. A. Gilles, J. K. Hamilton, P. A. Rebers, and F. Smith, Anal. Chem., 28, 350 (1956).

16. S. Okimasu, Bull. Agr. Chem., Soc. Jpn., 22, 63 (1958).

17. W. Yaphe and G. P. Arsenault, Anal. Biochem., 13, 143 (1965); T. Akahane, in preparation.

18. A. S. Cerezo, J. Chem. Soc. Sect. C, 992 (1967). 
19. A. S. Cerezo, J. Chem. Soc. Sect. C, 2491 (1967).

20. A. S. Cerezo, Carbohyd. Res., 18, 335 (1973).

21. T. J. Painter, Methods Carbohyd. Chem., 5, 98 (1965).

22. A. J. Pernas, O. Smidssød, B. Larsen, and A. Haug, Acta Chim. Scand., 21, 91 (1967).

23. G. A. Santos and M. S. Doty, J. Pharm. Sci., 64, 1704 (1975).
24. H. Eisenberg and R. Mohan, J. Phys. Chem., 63, 671 (1959).

25. K. Gekko and H. Noguchi, Macromolecules, 7, 224 (1974).

26. M. Duckworth and W. Yaphe, Carbohyd. Res., 16, 435 (1971). 